\title{
Key factors for determining the suitability of converting a fluid-mechanics laboratory to the remote-access mode
}

\author{
A. H. M. Faisal Anwar, Euan Lindsay and Priyantha Ranjan Sarukkalige \\ Curtin University, Perth, Australia \\ f.anwar@,e.lindsay@,p.sarukkalige@curtin.edu.au
}

\begin{abstract}
Remote laboratories are a mature technology that is becoming increasingly prevalent in the delivery of undergraduate engineering degree programs. While the literature has moved to the point where the educational value of these laboratories is being evaluated, there is presently no framework provided to help academics determine the suitability of a particular laboratory experiment for conversion to the remote-access mode. This paper analyses a second-year fluid-mechanics laboratory to determine its suitability for such a conversion, and then draws from this analysis three key factors that can be used as a preliminary framework for making generalised decisions regarding the suitability of any laboratory class for use in the remote mode.
\end{abstract}

\section{Introduction}

Laboratory classes are an essential part of the education of undergraduate engineers. Laboratories provide the opportunity to acquire a range of skills and knowledge that are not available through other avenues (Feisel \& Rosa, 2005). Providing these opportunities can be very expensive in terms of equipment and consumable costs, as well as the time and energy of academic staff required to prepare, supervise and assess these laboratories. As the size of engineering cohorts has grown, providing laboratory experiences to all students has become more challenging, with purchasing more and more equipment no longer a feasible solution.

One alternative solution is to provide web-based remote access to laboratory hardware. Remote access to the hardware can relax many of the constraints of the in-person experience - scheduling, supervision and directness of control can all be achieved much more easily when students can connect remotely via the internet, rather than requiring synchronised attendance in a physical laboratory

Remote laboratories were first introduced in 1996 (Aktan, Bohus, Crowl, \& Shor, 1996), and since then remote laboratories have become a relatively mature technology. The field has developed to the point where the literature contains reviews of remote laboratories (Ma \& Nickerson, 2006), and the challenges have moved from technical implementation through to pedagogical design and frameworks for inter-institutional sharing of equipment.

The focus of remote laboratory development is now moving towards more sustainable models. Rather than individual academics custom building equipment for their specialised subjects, remote laboratory development is increasingly being carried out by multi-institution consortia such as the Australian Labshare project (www.labshare.edu.au). These groups allow academics considering remote laboratories to take advantage of pre-existing tools to implement their experiments, rather than having to begin from scratch.

Even with this support for development, however, some kinds of equipment are more prevalent in the literature on remote laboratories. Topics dealing with control theory, such as PID or Programmable Logic Controllers, seem common. Simple mechanical systems like pendula or linked masses also appear often. The domination of the field by some kinds of laboratories raises questions. Are these laboratories more prevalent because they are better suited for remote conversion? Is there some combination of attributes of these experiences that makes them better suited for use in the remote 
mode? Or is it that the academics who teach these classes are more likely to be the kind of people who want to build remote laboratories?

There are pedagogical arguments to support the shift from an in-person experience to a remote-access mode, and the literature contains a number of references to the evaluation of student learning in the remote mode. The earliest evaluations, which only compared marks, found the modes to be equivalent for learning (Ogot, Elliot, \& Glumac, 2003), however more fine-grained evaluations have found differences in outcomes for the students. Lindsay \& Good (2005) showed that some learning outcomes can be enhanced through a transition to a remote mode, while other learning outcomes will be degraded. There are frameworks for evaluating the learning outcomes of remote laboratories eg, however the emerging consensus is that "direct comparison [between modes] is not appropriate or productive" (Hanson et al., 2009).

Depending on the balance of which outcomes are desired from a laboratory, it may be that remote access will provide a superior learning opportunity. To determine this, a range of factors - the type of learning required, the nature of the experiment, the willingness of the instructor - must be considered. By considering these factors, it should be possible to provide a metric for determining whether a particular laboratory experience is suitable for the remote-access mode, or whether it must be performed in the face-to-face environment.

The absence of such metrics from the literature is therefore somewhat startling. For all of the work that has been conducted on how to implement remote laboratories, there has been very little work on whether or not a laboratory is suitable. There has been evaluation work on the learning outcomes of the remote mode; however this is only one (admittedly very important) aspect of suitability for remote implementation.

This paper addresses the gap in the literature by using a case study at Curtin University to provide an evaluation framework and then generalises its contribution for use by others. The next section describes the existing face-to-face laboratory, while the third section describes the issues that affect its suitability for conversion to the remote access mode. The fourth section provides the preliminary generalisable framework, and the final section concludes the paper.

\section{The "Flow Through Pipes" Laboratory}

The flow through pipes laboratory is part of the second-year Fluid Mechanics 230 unit at Curtin University. This experiment allows students to explore how fluids act in different flow regimes (laminar, turbulent and transition), and to compare experimental measurements with the theoretically predicted model.

\section{The Experimental setup}

The equipment (Fig 1) consists of a $6.1 \mathrm{~m}$ long brass pipe through which oil is continuously circulated by a hydraulic pump. The pipe has nineteen piezometric tappings that are each connected to a mercury-filled manometer to measure the pressure at specified locations along the pipe. The flow in the pipe can be maintained laminar up to a Reynolds Number (Re) of about 2000. Turbulence is initiated by inserting a rod at the upstream end of the pipe; the flow becomes turbulent at an Re of about 5000. The pipe discharges into a transparent perspex catchment so that the difference between laminar and turbulent flow can be visually observed. 


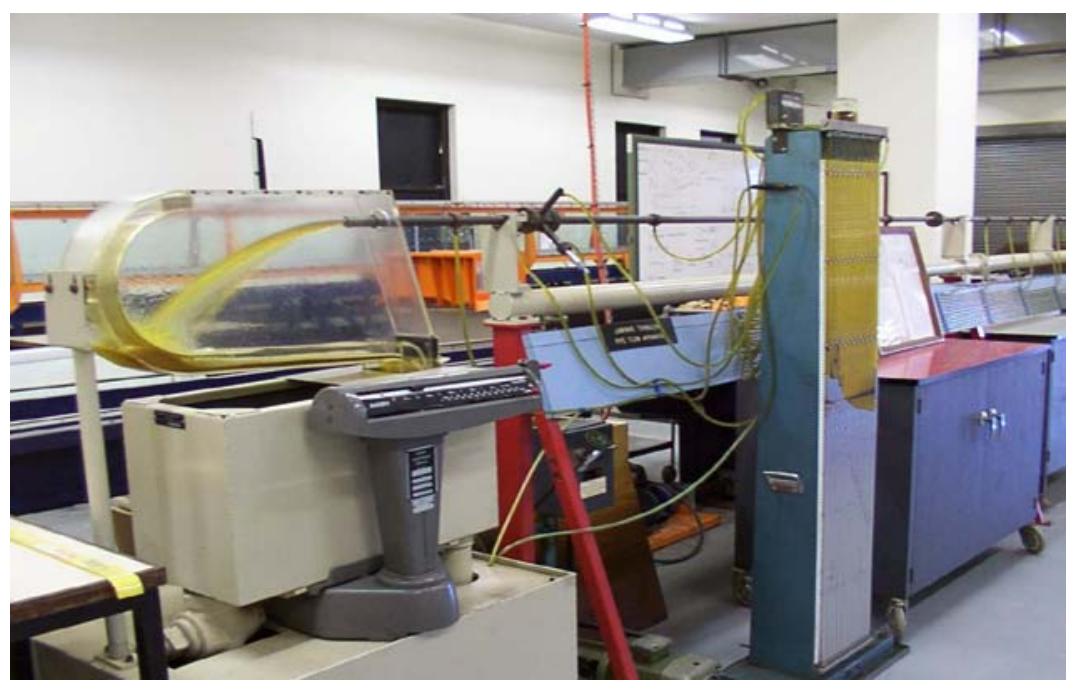

Fig. 1. Laboratory equipment of flow through pipe

The experimental rig is physically very large, and there is insufficient space within the laboratory to simply build multiple identical copies to address the student demand. This is one of the motivations for the consideration of remote access alternatives, provided the experiment proves suitable for conversion.

\section{Laboratory activities and learning outcomes}

There are a range of learning outcomes that students are intended to achieve through this laboratory class. The experimental assignment aims to cover a comprehensive study of flow in pipes. Therefore, this experiment is designed to illustrate laminar, transitional (intermittently turbulent), and fully turbulent pipe flows, and to determine the conditions under which these types of flow occur (Fig 2).

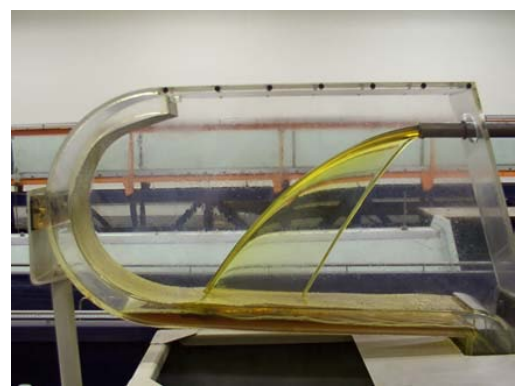

a

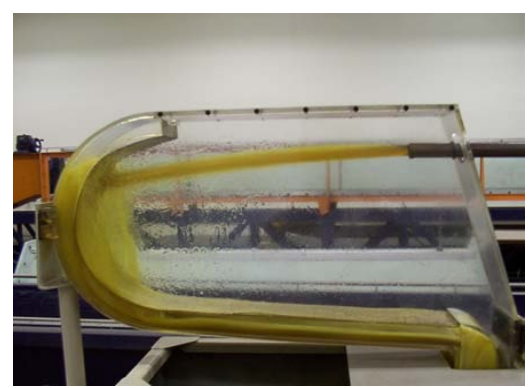

b

Fig 2. Typical (a) laminar flow and (b) turbulent flow

To explore these concepts, the students take a range of pressure and velocity readings along the pipe, allowing them to measure the pressure drops along, and velocity profile within, the pipe. These measurements, along with the student-controllable parameters of the flow (such as velocity) allow for the students to explore the nature of the flow. In particular, students are intended to understand the concept of friction applied to pipe flow, and to develop the relationships between the friction factor and the Reynolds number for laminar and turbulent flows. These measurements then allow for calculations to be made, and comparisons made between their results and the theoretical calculations learnt in the lectures.

In addition to these theoretically-driven objectives, the students are also expected to improve their professional skills as engineers. These include safe operation of the equipment, managing their time to complete all of the necessary tasks within the session, communicating with other students in their group, and managing their time to submit a report two weeks after conducting the experiment. 


\section{Suitability for Conversion to Remote Access}

The flow through pipes laboratory is being considered for conversion to a remote-access mode. Before this conversion can be made, however, it is crucial to consider the suitability of this experiment for implementation in this mode. This requires the analysis of a wide range of factors, each of which varies in the extent to which it supports the remote conversion.

\section{Visualisation of flow patterns}

One of the key objectives of this laboratory is for students to be able to visualize the different kinds of laminar and turbulent flow patterns, such as are shown in Fig 2. Conveying the visual elements of the experiment is well within the capabilities of a remote-access implementation. Indeed, depending upon the quality of the video cameras used, and of the monitors upon which the students view the video, it may be possible to provide a clearer view of the flow than would be possible inside a crowded laboratory.

It is also significant to note that while the flow patterns do depend upon the geometry of the experimental rig, they are not directly dependent upon the overall physical size of the apparatus. As such it will be feasible to construct smaller versions of the equipment for remote operation without the students losing access to the critical flow patterns.

\section{Laboratory scheduling}

Fluid Mechanics 230 is one of the largest units at Curtin University, with a total of more than 500 Civil, Mechanical and Chemical engineering students enrolled in the class. The flow through pipes laboratories are scheduled in the second half of the semester, after the relevant theory has been covered in lectures, leaving only a five-week window for all 500+ students to complete the laboratory on the same piece of equipment.

Ten, two-hour laboratory sessions are offered each week; this requires an average group size larger than ten. Reducing the size of these groups would require additional access time to the equipment; however there are significant difficulties in offering more than twenty hours a week of laboratory time, both in terms of demonstrator and student availability. Laboratory sessions cannot be scheduled to clash with other lectures or tutorials; with three different disciplines involved in the unit, this reduces the opportunities for additional laboratory time to be offered.

In addition to the structural difficulties in scheduling, there are also individual difficulties for students. Most students have commitments outside of their study; there are a constant stream of issues that arise from scheduling. Students miss their scheduled sessions for a variety of reasons, legitimate or otherwise. Some seek to avoid clashes by asking in advance to change their allocated session. During the five weeks the experiments are running, the laboratory coordinator for the unit receives an average of 5-10 emails a day from students seeking to make changes to their scheduled class.

Remote access to the laboratory hardware has the ability to significantly increase the amount of time for which the experiment is available. The equipment can be made available remotely $24 / 7$, allowing the students to schedule their access according to their own constraints, rather than requiring students to adapt to the university's timetable. Implementing multiple copies of the hardware will allow multiple students to access the equipment simultaneously, further easing the challenges of scheduling $500+$ students.

\section{Participation within the Laboratory}

It is inevitable with large group sizes that not all students can participate equally within the laboratory. This is partly due to a lack of opportunity - not everyone can control the equipment directly in the time allowed - but also due to the differences in students' learning styles and abilities. Students learn at different speeds; concepts come easier to some students than to others. 
The current scheduling arrangements require students to work in groups of ten or more, ensuring that there will be a range of learning speeds within the students. In this case it is almost inevitable that the group as a whole will be working either too fast or too slow for some of its members.

The dominant paradigm for remote access in the literature is an individual learning experience, in which single students control equipment. Working individually allows for each student to learn at their own pace - students who learn faster or slower than the average can take less or more time to achieve the same outcomes.

\section{Repetition of measurements}

Aside from the difficulty of fitting all of the students into a laboratory session, the time limitations also impact upon the way in which students learn within that session. One difficulty is that students are often not sure at the time of exactly what data they should be recording. While the theory gives them an indication of which parameters are important, it is often only when the students analyse the data after the laboratory that they realise which values of the parameters they should have considered. The current model provides no opportunity to take additional data to deepen the students' investigations; remote access allows for students to follow up and take more data after their initial session.

\section{Multiple Campuses}

In addition to the 500 students at Curtin's main Bentley campus, there are another 200 students enrolled in Fluid Mechanics 230 at Curtin's Miri Campus in Malaysia. The ability to service all 700 students with the same investment in laboratory hardware is an advantage.

\section{Variability of physical measurements}

One physical phenomenon that is demonstrated very well within the laboratory is the inherent variability of physical quantities. The friction losses in the pipes are measured through a series of mercury manometer readings. While the experiment is operational, the students can see the manometers flickering as the pressure in the pipe fluctuates, and can clearly relate this flickering to the variability of the flow in the pipe.

A remote conversion is unlikely to use an optical-inspection approach for measuring pressure; instead, some kind of pressure transducer will be used. There is a concern that this will impact on the students' understanding of the variability through two possible avenues. The first is that the transducer will report only an averaged pressure value, and that the variability will be hidden from the students. The second is that if the variability is conveyed, that it will be interpreted by the students as some kind of error in the interface, rather than as an accurate representation of the physical phenomenon.

\section{Remote Controllability}

The equipment has the advantage that it is relatively straightforward to convert to remote access. Each of the parameters that need to be measured - such as pressure and flow rate - can be measured using sensors that can be digitised easily. The quantities that must be controlled - such as pump speed - can be controlled through digitally-operated actuators. This would require some changes in the way in which quantities are measured, but it will not require major adjustments to the way in which the experiment is implemented.

\section{Tactile Experience}

Some elements of the laboratory experience cannot be replicated in a remote mode. While students can certainly see the different types of flow regimes through a video link up, and they can hear the changes in pump sounds as the flow rate increases through an audio link, there are some senses that cannot be conveyed remotely. The oil used in the experiment has a distinctive smell that becomes more prominent when the flow rate increases; similarly the laboratory itself has an ambience different to that of a lecture theatre or classroom.

The direct hands-on elements of the experiment are also impossible to replicate. While electronic commands can be used to choose a new setpoint for the flow rate, this differs from the students 
manually turning a stopcock to allow more fluid to flow. In the course of their professional careers it is more likely that they will operate valves remotely, rather than by hand, but it is still important that at some point in their training they understand the manual version. While not essential to this particular laboratory, it is an outcome that cannot be abandoned altogether.

\section{Key Factors for determining suitability - a Generalised framework}

Overall, the flow through pipes laboratory is suitable for conversion to remote access. This decision was made after considering which factors led to improvements, which led to compromises, and which remained neutral. When aggregating the analysis, the factors fell into three generalisable categories, which can also be used as a generalised framework for considering other laboratories: Learning factors, Equipment factors and Cohort factors.

\section{Learning factors}

Ultimately, the most important question is whether the students would learn as well or as much from the remote implementation. Analysing the options shows that the core elements of the laboratory can be learned just as well in a remote implementation, and in some instances the remote access will potentially allow for improvements in student learning. Increased access time and self-paced access offers an opportunity to allow the students to learn in the way they wish, rather than the way in which the group they are allocated to chooses as its norm.

It is unavoidable that some learning outcomes cannot be achieved as well (or at all) in the remoteaccess mode. Direct hands-on control of the equipment is impossible; however when compared to understanding the differences between turbulent and laminar flow, it is less important.

When aggregated, the learning factors for the laboratory come out essentially neutral; students can meet the core learning objectives equally well in either mode.

\section{Equipment factors}

The equipment in this experiment is intended for illustrating the theoretical concepts; it is not essential that the students develop skills with this exact hardware. In other laboratories students are expected to develop proficiency in the use of an oscilloscope, or a soldering iron, or another piece of laboratory kit. In this laboratory, however, the equipment itself is unimportant, except as a method for illustrating the concepts involved.

This experiment is well suited for conversion to a remote-access mode. It is a closed loop system, that does not require the user to insert or remove workpieces or samples. The sensors can be converted for remote access using off-the-shelf components, as can the actuators involved.

\section{Cohort factors}

The cohort of students who use this experiment make it suited for remote access. The large numbers of students, combined with the relatively short window in which they all must gain access to the equipment, support the drive to make the experiment available in more flexible ways.

\section{Conclusion}

This paper has introduced a high-demand laboratory class in fluid mechanics, and has analysed this experiment for suitability for conversion to remote access. There are some learning outcomes that will not be able to be achieved as well in the remote mode, there are some that may be enhanced while others remain neutral to the delivery mode. This overall neutrality of learning outcomes allows for the logistical considerations to dominate the decision, and it is clear by the analysis that remote access has the potential to greatly relax the significant scheduling constraints of the existing lab.

In addition to this analysis, this paper draws from the analysis three generalisable factors for making judgements concerning a laboratory's suitability for conversion: learning factors, equipment factors 
and cohort factors. In this scenario, all three factors are either positive or neutral regarding the conversion, indicating clearly the value of a remote conversion. In situations where these factors may be contradictory, a more sophisticated and detailed framework would be warranted.

\section{References}

Aktan, B., Bohus, C. A., Crowl, L. A., \& Shor, M. H. (1996). Distance Learning Applied to Control Engineering Laboratories. IEEE Transactions on Education, 39(3), 320-326.

Feisel, L. D., \& Rosa, A. J. (2005). The Role of the Laboratory in Undergraduate Engineering Education. Journal of Engineering Education, 94(1), 121-130.

Hanson, B., Culmer, P., Gallagher, J., Page, K., Read, E., Weightman, A., et al. (2009). ReLOAD: Real Laboratories Operated at a Distance. Transactions on Learning Technologies 2009, 2.

Lindsay, E. D., \& Good, M. C. (2005). Effects of Laboratory Access Modes Upon Learning Outcomes. IEEE Transactions on Education, 48(4), 619-631.

Ma, J., \& Nickerson, J. V. (2006). Hands-On, Simulated, and Remote Laboratories: A Comparative Literature Review. ACM Computing Surveys, 38(3).

Mohtar, A., Nedic, Z., \& Machotika, J. (2008). Evaluating the Effectiveness of a Remote Laboratory for Microelectronics Fabrication. Paper presented at the AAEE 2008: 19th Annual Conference, Yeppoon, Australia.

Ogot, M., Elliot, G., \& Glumac, N. (2003). An assessment of In-Person and remotely operated laboratories. Journal of Engineering Education, 92(1), 57-63.

\section{Acknowledgements}

The authors acknowledge the funding support of DEEWR's Diversity and Structural Adjustment Fund through their grant "National support for Laboratory Resource Sharing". 